\title{
Orbital Period Modulation in SW Cygni
}

\author{
Davood Manzoori ${ }^{\mathrm{A}}$ \\ A Department of Physics, Mohaghegh Ardabili University, P.O. Box. 56199-11367, Ardabil, Iran. \\ Email: d.manzoori@uma.ac.ir
}

Received 2006 December 19, accepted 2007 May 26

\begin{abstract}
The O-C curve of the Algol-type eclipsing binary SW Cyg was analyzed using the Kalimeris method. The observed period variations with time, the $P(E)$ function and its rate of change $\mathrm{d} P / \mathrm{d} E$, were calculated. The plots of $\mathrm{O}-\mathrm{C}$ values as well as of $P(E)$ and $\mathrm{d} P / \mathrm{d} E$ against ephemeris $(E)$ all show rather regular period variations in this system. To reveal any cyclic period variations, the $P(E)$ function was subjected to Fourier analysis. A cyclic period change of average $\approx 27.8$-yr duration was obtained. Also a relative mass transfer rate of $\Delta m / m=-1.1 \times 10^{-9} \mathrm{yr}^{-1}$ was estimated. Finally the existence of a third companion is suggested, and possible causes of period variations in the system are discussed.
\end{abstract}

Keywords: Stars: Binaries eclipsing — Stars: individual — SW Cyg

\section{Introduction}

The binary system SW Cyg (HD 191240, BD +453062) has period $P=4.573 \mathrm{~d}$ and spectral type $\mathrm{A} 2 \mathrm{eV}+\mathrm{K} 0 \mathrm{IV}$. Blazko (1924) was the first who noted the period variations of the system. Recent works dealing with period variations of the system are as follows:

Walter (1971) was the first who studied the system photometrically in $B$ and $V$ pass-band filters. He reported an asymmetric primary minimum and fluctuating additional light, the origin of which was detected to be on the A star (i.e. hotter component). Also the deviation between calculated and observed light curves was interpreted by additional light on the primary.

Hall \& Garrison (1972) made $U B V$ photometry of the system. In addition to the asymmetries at primary eclipse, they found variations of luminosity, and excess of UV radiation from the cool subgiant, which they attributed to contamination by light from an envelope surrounding the hot star and not completely eclipsed at mid primary minimum.

Frieboes-Conde \& Herczeg (1973) reported sudden, discontinuous period variations, and distorted sine curves for $\mathrm{O}-\mathrm{C}$ values (i.e. figures 12 and 13 in their paper) with period $P \approx 80 \mathrm{yr}$ a possible evidence of an invisible third body with mass $m_{3}=8 \mathrm{M}_{\odot}$ which was suggested to be a black hole. An important phenomenon reported by them with certainty was irregular brightness fluctuations during totality. This phenomenon was also observed earlier by Wendell (1909), Walter (1971), and Hall \& Garrison (1972).

Olson \& Etzel (1993) made five-color intermediate band photometry of totality in the SW Cyg system. They found small brightness fluctuations of the cool subgiant star (a common phenomenon to almost all Algols which were under their investigation). This brightness variation was explained in terms of star-spot activity and area.
Chauby (1980) analyzed the O-C curve, he also reported the mass transfer and a third body as the causes of period change in this system.

Berrington \& Hall (1994) calculated and discussed the presence of a third body with mass $m_{3}=10 \mathrm{M}_{\odot}$. Because of the rapid rotation of the secondary component, and its possession of a convective layer, they preferred to suggest modulation of period through a cycle of magnetic activity (of duration 96 years) in the secondary component as the cause period variation, rather than a third body.

In their study of period changes of SW Cyg, Todoran $\&$ Agerer (1994) (using the uncertain eccentricity $e=0.3$ ) also calculated the mass of a possible third body, i.e. $m_{3}=10.56 \mathrm{M}_{\odot}$, (probably a black hole as they mentioned), but they also reported apsidal motion as a main likely cause of period variability.

Simon (1997) studied period variations of SW Cyg. From the analysis of the O-C curve and comparison of the obtained results with those of Olson \& Etzel (1993) and Olson (1985), he inferred the existence of a correlation between variations of orbital period and changes of the level of totality in SW Cyg. Moreover, he found that episodes of decrease of the period are accompanied by minima of the brightness in the secondary component, this fact indicate that period change may be occurring due to instabilities in the outer convective layer of secondary gaint.

Qian, Zhu, \& Boonrucksar (2002) have suggested that active mass transfer is an effective factor causing period change in SW Cyg. They also found several random jumps in the period which led them to completely ignore the role of a third body in the period variations.

In the present work, to study period variations of SW Cyg we plotted the $\mathrm{O}-\mathrm{C}$ differences against ephemeris $E$, and a tenth-order polynomial was best fitted to the 
points (Section 3.1). Applying a method introduced by Kalimeris, Livaniou, \& Rovithis (1994), we used the above polynomial coefficients to construct $P(E)$, i.e. the change of period with time, and $\mathrm{d} P / \mathrm{d} E$, the time rate of change of $P(E)$ (Section 3.2). Finally to detect the existence of cyclic period changes (if any), we subjected the $P(E)$ function to Fourier analysis. The frequency spectrum of the analysis is discussed in Section 3.3.

\section{Causes of Period Variations}

The accuracy obtainable in period determination and changes of period has provided a unique tool to study stellar structure, geometry and evolution. The gravitational and magnetic interactions of individual stars and the region in between the components of a close binary system can lead to orbital period variation. These variations manifest themselves in the $\mathrm{O}-\mathrm{C}$ curve. There are various causes of period variations in different systems, as briefly explained below:

- Frequently the O-C curve is periodic and can be fitted by a sine curve. In such cases, the most probable causes are:

1. Apsidal motion. This demands an eccentric orbit and if the time residuals of secondary minimum are plotted against $E$, the ephemerides, the resulting curve shows the same form but in opposite phase with respect to primary curve.

2. The presence of a third body in an orbit with system. Except for strictly periodic changes, this often requires an extremely large elliptic orbit.

- Period variations have been mostly observed in systems in which at least one component is a late F-K type star. In these types of stars the appearance of magnetic activity is a common phenomenon (Hall 1989; Richards \& Albright 1993). And since actually the eclipsing binaries of Algol type possess such characteristics, many authors (e.g. Applegate \& Patterson 1987; Applegate 1989; Hall 1989, 1991; Olson \& Etzel 1993; Lanza, Rodono, \& Ronse 1998) who have studied period variations in the eclipsing binary stars have suggested that the modulation of period through a magnetic activity cycle variation is the main cause of period variability in Algols.

- The most plausible mechanism, which has been put forward by Applegate (1992), and modified by Lanza et al. (1998), was based on the variation of the active star's shape through change of the gravitational quadrupole moment (GQM) of the star. This change in GQM is coupled to the orbit, producing orbital period changes as follows: The GQM is most sensitive to the rotation rate of the outer parts of the star. Because of magnetic torque exerted on the outer parts of the star, the distribution of angular momentum and as a result the oblateness of the star changes as the star goes through activity cycles. These rotation rate and oblateness changes will be communicated to the orbit by gravity, leading to orbital

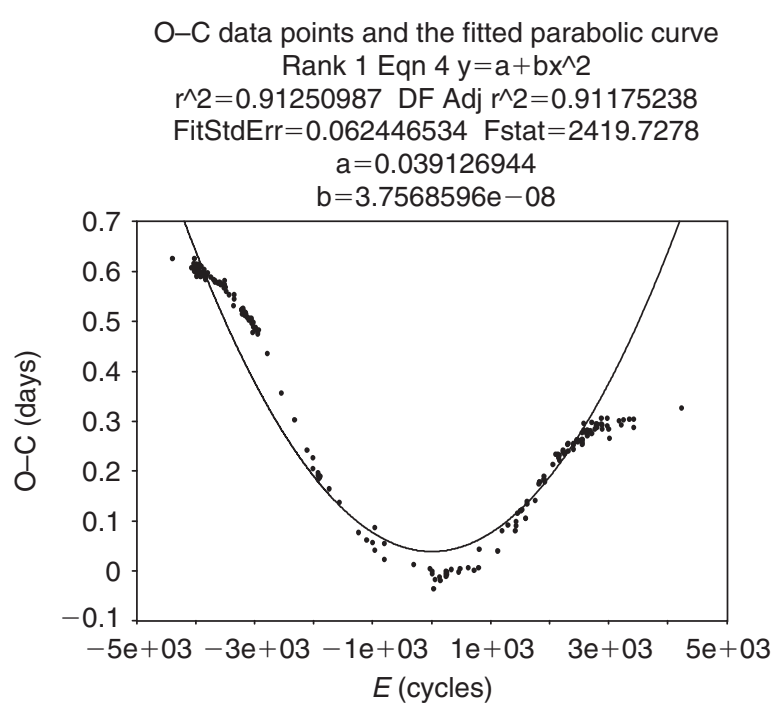

Figure 1 The $\mathrm{O}-\mathrm{C}$ diagram (dots) and least-squares quadratic (parabolic) fit (continuous curve) for SW Cyg.

period variations. One requirement for this mechanism is luminosity variation of the active star with the same period as the orbital period modulation. The rotational kinetic energy increases during one half of the cycle, because of angular momentum transfer by magnetic torque, and, since energy is absorbed from convective heat flow, the star is driven away from solid-body rotation. During the other half cycle the action takes place in the reverse order. The resulting effect will be a fluctuation of luminosity. This variation of luminosity depends on the how fast the convective zone of the active star responds to variations of heat flow.

- Mass and angular momentum loss and/or transfer from the Lagrangian point $\mathrm{L}_{2}$ (Rocket effect), involving magnetic braking (Hall \& Kreiner 1980; Chaubey 1984).

- Mass motion in the interior of one or both components.

- Large-scale main circulation within the system in a common envelope (Hack 1984).

\section{O-C Curve Analysis}

To study the period variations of this system, I have collected timings of minima from different sources up through July 2006, mainly from Berrington \& Hall (1994) and other available sources (e.g. IBVS No. 5399, 5643, and BBSAG Bulletin No. 113,110, 107). The collected data were reduced to a common epoch according to the ephemeris given by Frieboes-Conde \& Herczeg (1973), Equation (1):

$$
\operatorname{Min}(\mathrm{HJD})=2433160.669+4.5729841 E
$$

\subsection{Least-Squares Curve Fitting}

The $\mathrm{O}-\mathrm{C}$ values were calculated and plotted against ephemeris $E$. The general trend of $\mathrm{O}-\mathrm{C}$ values displayed in Figure 1 shows an upward curved parabola which can be roughly fitted by the following quadratic equation:

$$
\mathrm{O}-\mathrm{C}=0.04398895+3.6903544 E^{2}
$$


This overall quadratic character of $\mathrm{O}-\mathrm{C}$ curve suggests a secular period increase for the system. Figure 2 displays the residuals of quadratically fitted parabola from the $\mathrm{O}-\mathrm{C}$ values, the wave form of these residual points suggests the presence of some alternately changing sign mechanisms are acting on the system.

Further more to interpret the $\mathrm{O}-\mathrm{C}$ curve more accurately, a tenth-order polynomial was best fitted to the $\mathrm{O}-\mathrm{C}$ data points, as shown in Figure 3 , and the coefficients, $c_{j}$, of the polynomial along with root mean square (rms) errors and correlation coefficient $r^{2}$ are listed in Table 1. The residuals of $\mathrm{O}-\mathrm{C}$ values from the fitted polynomial are indicated in Figure 4. As can be seen, they do not deviate more than $\approx 0.02$ days, which is within the observational errors. These residual points are quite scattered and show no oscillatory term, signifying that any modulation of the period has already been described by the mentioned 10th-order polynomial.

\subsection{The $P(E)$ Function}

The Kalimeris et al. (1994) method has been used to calculate the observed period changes $\left(P-P_{\mathrm{e}}\right.$, where $P_{\mathrm{e}}$ is the ephemeris period) and time rate of period change functions $\mathrm{d} P / \mathrm{d} E$ through the Equations (3) and (4) below:

$$
P(E)=P_{e}+\Delta T(E)-\Delta T(E-1)
$$

While the rate of change of period at any cycle $E$ is given by:

$$
\begin{aligned}
\dot{P}(E)=\frac{\mathrm{d} P}{\mathrm{~d} E}= & \frac{1}{c}\left(\sum_{j=0}^{n-1}(j+1) c_{j+1} E_{N}^{j}\right. \\
& \left.-\sum_{j=0}^{n-1}(j+1) c_{j+1}\left(\frac{E-1}{c}\right)^{j}\right)
\end{aligned}
$$

And the period change per cycle i.e. $E_{1}$ and $E_{2}$ as

$$
\begin{aligned}
\Delta P(E)= & \int_{E_{1}}^{E_{2}} \dot{P}_{E} \mathrm{~d} E=\Delta T\left(E_{2}\right)-\Delta T\left(E_{2}-1\right) \\
& +\Delta T\left(E_{1}\right)-\Delta T\left(E_{1}-1\right)
\end{aligned}
$$

which, for two successive cycles $\Delta E=E_{2}-E_{1}=1$, gives the period change per cycle.

Both of these functions, i.e. $P(E)$ and $\mathrm{d} P / \mathrm{d} E$ are depicted in Figure 5. As can also be seen visually from Figure 5, the $P-P_{\mathrm{e}}$ curve shows a wave like character, and at least five extremes are evident. Moreover it is clear from the extended cycle between the years 1885 and 2003, that there is a change of $\Delta P$ phase from negative to positive (i.e. decrease to increase of $P$ ). The cyclic variations, and an almost constancy of $P$ around the year 1940, are also evident from the $\mathrm{d} P / \mathrm{d} E$ curve of Figure 3 .

As may be seen from the Figure 6 the $P(E)$ function can be approximated relatively good by a sine wave (or we can say that the $P(E)$ curve is a distorted sine wave). The residuals of $P(E)$ function from the approximated sine wave have been displayed in Figure 7 . The cyclic effects 
Residuals of fitted parabolic curve from the $\mathrm{O}-\mathrm{C}$ values Rank 1 Eqn $4 \mathrm{y}=\mathrm{a}+\mathrm{bx} \wedge 2$ $\mathrm{r}^{\wedge} 2=0.91250987$ DF Adj $\mathrm{r}^{\wedge} 2=0.91175238$ FitStdErr $=0.062446534$ Fstat $=2419.7278$ $\mathrm{a}=0.039126944$ $b=3.7568596 e-08$

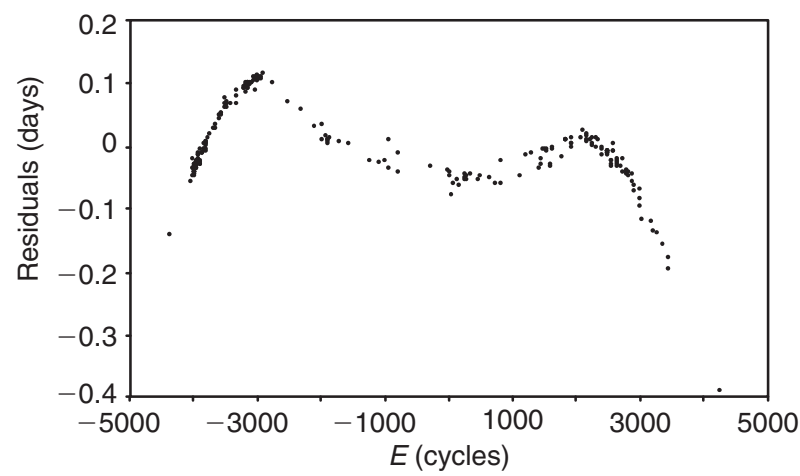

Figure 2 The residuals between the observed $\mathrm{O}-\mathrm{C}$ values and the fitted parabola.

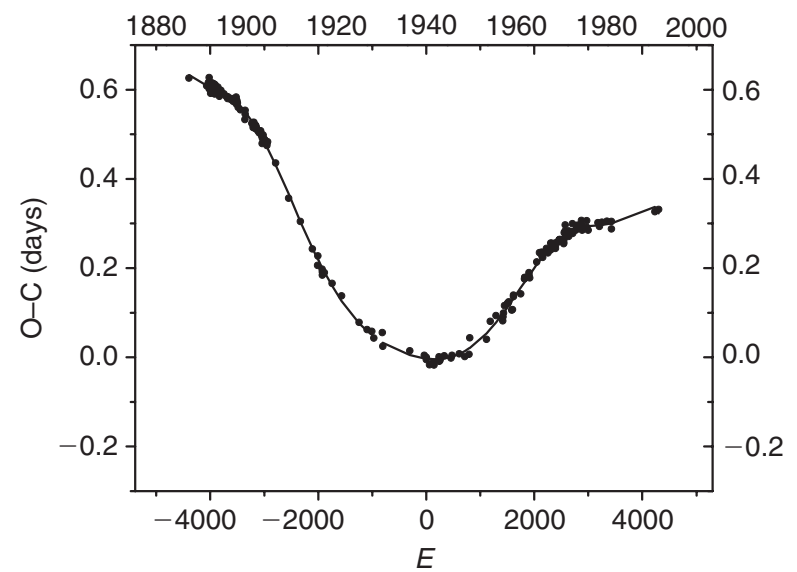

Figure 3 The $\mathrm{O}-\mathrm{C}$ diagram (dots) and its description by a least-squares polynomial (continuous curve) for SW Cyg.

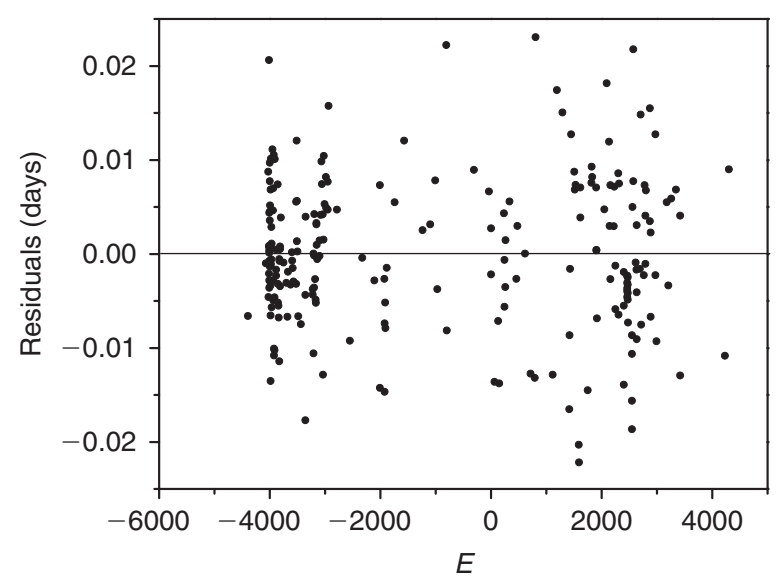

Figure 4 The residuals between the observed $\mathrm{O}-\mathrm{C}$ differences and the best fitted polynomial $\Delta T(E)$.

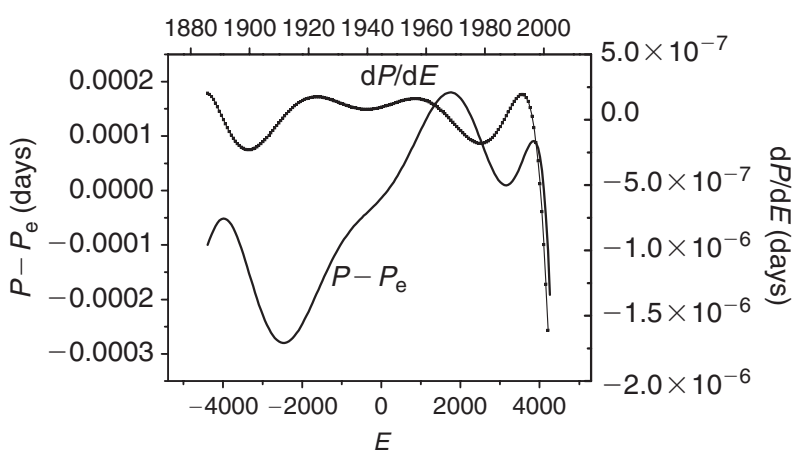

Figure 5 The upper curve indicates the rate of period change $(\mathrm{d} P / \mathrm{d} E)$, and the lower one shows the period of SW Cyg as a function of time. The difference $P-P_{\mathrm{e}}$ is referred to the ephemeris period $P_{\mathrm{e}}=4.5729841$.

$$
P-P_{\mathrm{e}} / E
$$

Rank 1 Eqn 8015 SineSquared (a,b,c,d) $\mathrm{r}^{\wedge} 2=0.88758004$ DF Adj $\mathrm{r}^{\wedge} 2=0.88216221$ FitStdErr $=4.6962022 \mathrm{e}-05$ Fstat $=221$

$a=-0.00022318522 b=0.00037392667$ $c=4.0208211 \mathrm{~d}=16681.791$

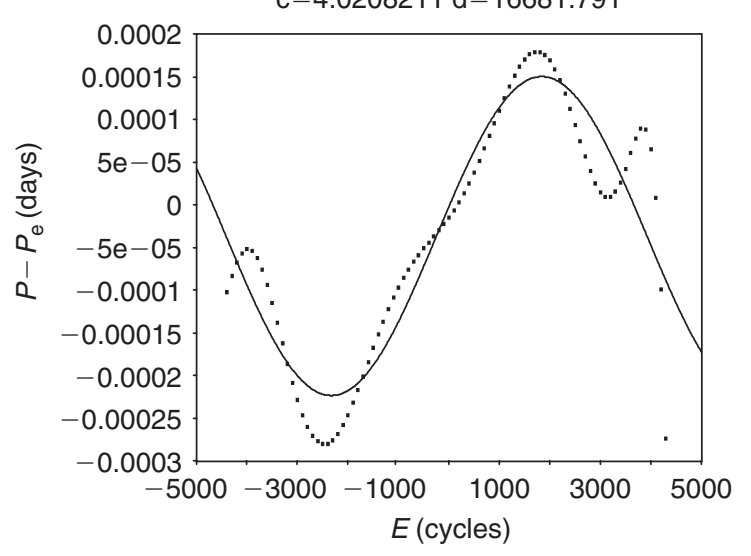

Figure 6 The $P(E)$ function (dots) and its approximation by a sine wave (continuous curve) for SW Cyg.

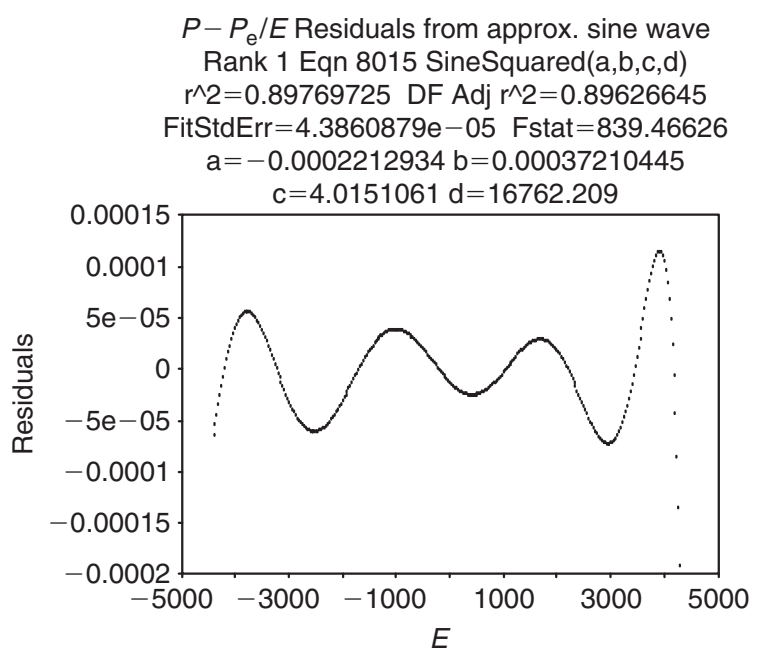

Figure 7 The residuals between the $P(E)$ function and approximated sine wave. 


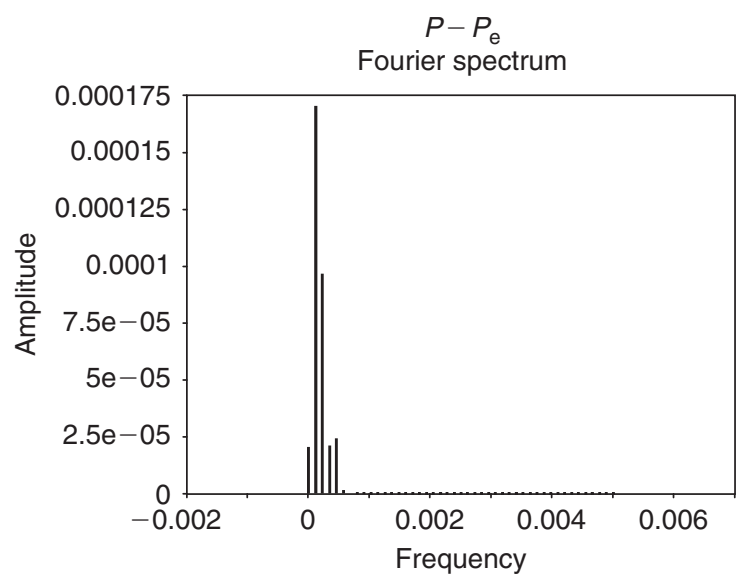

Figure 8 The frequency spectrum of the $P(E)$ function of SW Cyg obtained through Fourier analysis.

Table 2. Interpolated spectral peaks

\begin{tabular}{lcc}
\hline Frequency & Amplitude (days) & Period (yr) \\
\hline 0.00012272 & 0.00017193 & $102.02 \pm 7.4$ \\
0.00044924 & $2.4687 \mathrm{e}-05$ & $27.87 \pm 2.38$ \\
\hline
\end{tabular}

are again quite clear from this figure after subtraction of the sine wave. Alternately changing sign and wave like behavior of the $\mathrm{d} P / \mathrm{d} E$ (Figure 5) function confirms the above results.

To reveal any periodic phenomena present in The $P(E)$ function it was subjected to Fourier analysis, the frequency spectrum of which is shown in Figure 8. In the frequency spectrum of $P(E)$ function obtained (Figure 8) there are two distinct peaks well above the noise level with particulars listed in Table 2.

\subsection{The Third Mass}

We attribute the first peak of the Fourier spectrum (i.e. a long cycle of $102.02 \pm 7.4-y r$ duration) to motion of a third mass around the system (see the Section 4.2 discussion). The radius of the orbit and the mass of the third body may be estimated by using Equations (6) and (7) below:

$$
\begin{aligned}
2 a & \sin (i)\left(\sqrt{1-e^{2} \cos ^{2} \omega}\right. \\
\quad=c & {\left.\left[(\mathrm{O}-\mathrm{C})_{\max }-(\mathrm{O}-\mathrm{C})_{\min }\right]\right) }
\end{aligned}
$$

(given by Irwin 1959), and the well-known equation of mass

$$
F(m)=\frac{a^{3}}{P^{2}}=\frac{m_{3}^{3}}{\left(m_{1}+m_{2}+m_{3}\right)^{2}}
$$

where

$c=2.592 \times 10^{10} \mathrm{~km} /$ day, the velocity of light,

$i=78^{\circ}$, the orbital inclination,

$\omega=20^{\circ}$, the longitude of periastron,

$a=$ orbital radius, $m_{1}=2.8 \mathrm{M}_{\odot}$, mass of the hot component (primary),

$m_{2}=0.7 \mathrm{M}_{\odot}$, mass of the cool component (secondary),

$e=0.11$, eccentricity,

$P_{1 \text { mod }}=102.02 \mathrm{yr}$, modulating period,

and $(\mathrm{O}-\mathrm{C})_{\max }-(\mathrm{O}-\mathrm{C})_{\min }=0.61$ days, the full amplitude of $\mathrm{O}-\mathrm{C}$ curve obtained from Figure 1 ,

have been used to estimate the possible third mass $m_{3}=19 \mathrm{M}_{\odot}$ and the corresponding orbital radius $a=52.1$ a.u. Although the appearance of the $\mathrm{O}-\mathrm{C}$ curve shows the existence of approximately the same period as obtained from the first peak of the Fourier spectrum, this peak should be treated with little certainty because of the possibility of superposition of the time span of all data points on that frequency (i.e. $f_{1}=0.00012272$ ).

\subsection{Mass Transfer}

In an interacting binary system where the cool secondary component (donor) is an evolved star and fills its Roche lobe, mass transfer is expected from the Lagrangian $L_{1}$ point of the system, i.e. the stream of gas (with the rate of $\Delta m=-7.56 \times 10^{-10} \mathrm{M}_{\odot} \mathrm{yr}^{-1}$ as estimated below in this work) accelerates towards hot primary through a mechanism given by Bondi \& Hoyle (1944). Assuming a conservative case (i.e. when no matter leaves the system) and using the relative period rate of change $\triangle P / P=2.43 \times 10^{-9}$ obtained in this work and the equation:

$$
\frac{\Delta m_{2}}{m_{2}}=-\left(\frac{m_{1}}{3\left(m_{1}-m_{2}\right)}\right) \frac{\Delta P}{P}
$$

given by Huang (1963), a relative mass transfer of $\Delta m_{2} / m_{2}=-1.1 \times 10^{-9}$ and hence the corresponding mass and angular momentum lost by secondary were obtained as $\Delta m_{2}=-7.56 \times 10^{-10} \mathrm{M}_{\odot} \mathrm{yr}^{-1}$ and $\Delta J=-3.7 \times 10^{47} \mathrm{~g} \mathrm{~cm}^{2} \mathrm{~s}^{-1}$, respectively. We have used the masses of the components given by Hall \& Garrison (1972).

\subsection{Estimation of Some Parameters to Check the Applegate Theory}

Mass transfer and cyclic magnetic activity in the Algol type binary stars (particularly the secondary component) would cause the orbital parameters like $J$ (orbital angular momentum), $a$ (orbital radius) and $\Omega$ (angular speed) to change accordingly. Here we follow Applegate (1992), Lanza et al. (1998), and Kalimeris et al. (1994) to estimate some of these quantities for a conservative case as below:

$$
\begin{aligned}
\Delta J & =-\left(\frac{G m_{2}^{2}}{R}\right)\left(\frac{a}{R}\right)^{2} \frac{\Delta P}{6 \pi} \\
\Delta E^{\prime} & =\Omega_{\mathrm{d} r} \Delta J+\frac{(\Delta J)^{2}}{2 I_{\mathrm{eff}}} \\
\Delta L_{\mathrm{rms}} & =\pi \frac{\Delta E}{P_{\mathrm{mod}}}
\end{aligned}
$$


Table 3. The estimated parameters corresponding to modulation period 27.87

\begin{tabular}{lc}
\hline Parameter & $P_{2 \text { mod }}=27.87(\mathrm{yr})$ \\
\hline$\Delta J\left(\mathrm{~g} \mathrm{~cm}^{2} \mathrm{~s}^{-1}\right)$ & $-3.7 \times 10^{47}$ \\
$\Delta E(\mathrm{erg})$ & $1.40 \times 10^{40}$ \\
$\Delta L_{\mathrm{rms}}\left(\mathrm{erg} \mathrm{s}^{-1}\right)$ & $4.90 \times 10^{31}$ \\
$\frac{\Delta \Omega}{\Omega}$ & $8.57 \times 10^{-4}$ \\
$\Delta Q\left(\mathrm{~g} \mathrm{~cm}^{2}\right)$ & $-2.34 \times 10^{51}$ \\
$B(\mathrm{KG})$ & 1.27 \\
$\mathrm{~d} a / \mathrm{d} E\left(\mathrm{~cm} \mathrm{cycle}^{-1}\right)$ & 0.16 \\
$\mathrm{~d} \Omega / \mathrm{d} E\left(\mathrm{rad} \mathrm{cycle}^{-1}\right)$ & $-3.17 \times 10^{-17}$ \\
\hline
\end{tabular}

$$
\begin{aligned}
\frac{\Delta \Omega}{\Omega} & =\frac{G M^{2}}{3 R^{3} m_{\mathrm{s}}}\left(\frac{a}{R}\right)^{2}\left(\frac{P}{2 \pi}\right)^{2} \frac{\Delta P}{P} \\
\Delta Q & =-\left(\frac{M R^{2}}{9}\right)\left(\frac{a}{R}\right)^{2} \frac{\Delta P}{P} \\
N & \sim \frac{B^{2}}{4 \pi}\left(4 \pi R^{2}\right) \Delta R \sim 0.1 B^{2} R^{3} \\
B^{2} & \approx 10 \frac{G M^{2}}{R^{4}}\left(\frac{a}{R}\right)^{2}\left(\frac{\Delta P}{P_{\mathrm{mod}}}\right) \\
\frac{\mathrm{d} a}{\mathrm{~d} E} & =\left[\frac{2 G\left(m_{1}+m_{2}\right)}{27 \pi^{2}}\left(\frac{1}{P}\right)\right]^{1 / 3} \frac{\mathrm{d} P}{\mathrm{~d} E} \\
\frac{\mathrm{d} \Omega}{\mathrm{d} E} & =\left(\frac{2 \pi}{P^{2}}\right) \frac{\mathrm{d} P}{\mathrm{~d} E}
\end{aligned}
$$

where $a / R=3.12$, in which $R$ is radius of the secondary component, $\Delta P=2.132956800 \mathrm{~s}$ are the orbital period change during $27.87 \pm 2.38$-yr cycles respectively, $\Delta E^{\prime}$ is energy required to transfer angular momentum $\Delta J$, $m_{2}$ and $m_{\mathrm{s}}$ are masses of the secondary component and surrounding shell respectively, $I_{\mathrm{S}}$ and $I_{*}$ are moments of inertia of the surrounding shell and star respectively so that $I_{\mathrm{S}} \approx I_{*} \approx 2 I_{\mathrm{eff}}$, and $I_{\mathrm{eff}}=I_{\mathrm{S}} I_{*} /\left(I_{\mathrm{S}}+I_{*}\right), \Delta Q$ is the gravitational quadrupole moment of the active star, $B$ is the magnetic induction field, and $\mathrm{d} a / \mathrm{d} E$ and $\mathrm{d} \Omega / \mathrm{d} E$ are the rates of change of orbital radius and orbital speed, respectively. Using the above equations, i.e. Equations (9)-(19), and modulating period $P_{2 \mathrm{mod}}=27.87 \mathrm{yr}$, we obtained the following quantities listed in Table 3.

The values estimated above (in Table 3 ) are in accordance with corresponding estimated values by Applegate (1992) for Algol and other systems.

To compare the results of Applegate and Lanza, we used the equations (18) and (19) below borrowed from Lanza (1998):

$$
\begin{gathered}
\Delta Q=\left(\frac{-M a^{2}}{9}\right) \frac{\Delta P}{P} \\
\frac{\Delta \Omega}{\Omega}=\left(\frac{9}{8 K_{2}} \frac{G}{R^{5} \Omega^{2}}\right) \Delta Q
\end{gathered}
$$

where $K_{2}$ is the apsidal motion constant; to estimated values of $\Delta Q=-2.34 \times 10^{51} \mathrm{~g} \mathrm{~cm}^{2}$ and $\Delta \Omega / \Omega=6.75 \times$
$10^{-3}$, these values were found to be in fair agreement with the corresponding values calculate from Equations (12) and (13) above.

\section{Discussion and Conclusions}

Visual inspections of the observed $\mathrm{O}-\mathrm{C}$ points and the fitted curve depicted in Figure 1, and cyclic semi regular form of the residuals points of fitted parabola from the $\mathrm{O}-\mathrm{C}$ values (Figure 2), followed by subsequent analysis of the $P-P_{\mathrm{e}}$ curve for $\mathrm{SW} \mathrm{Cyg}$, show relatively regular cyclic variations. In such cases probable causes of period changes are either apsidal rotation or a third body in orbit with the system.

\subsection{Apsidal Motion}

Apsidal motion requires an eccentric orbit and requirements of the third body are extremely large and a regular orbit (as mentioned in Section 2). Todran \& Agerer (1994) used eccentricity a value of $e=0.3$ (obtained by Struve 1946) to calculate apsidal constants for their apsidal motion hypothesis. But this value of $e$ was not a sure value, as stated by them and also by other investigators, e.g. Frieboes-Conde \& Herczeg (1973) who argued that because of a biasing effect of the gas stream from the secondary cool star on the radial velocity curve of SW Cyg, the spectroscopic data of Struve which were used to estimate orbital eccentricity $e=0.3$ was not a significant one, and they suggested $e=0.11$ instead. Chauby (1980) calculated $e=0.01$ and phase of secondary minimum $\sim 178^{\circ}$ for the year 1964, which was in fair agreement with minimum phase calculated through photometric observations (i.e. $180^{\circ}$ ) by Walter for the same year, therefore primary and secondary minima were in phase. Unfortunately, I could not locate enough secondary minimum timings, i.e. $(\mathrm{O}-\mathrm{C})_{2}$ values, in the literature which could help to make a strict decision in this regard, so photometric and spectroscopic observations of the system strongly are suggested. On the basis of the above discussion, I believe that the possibility of the apsidal motion to be responsible for the period variation in this system is almost ignorable.

\subsection{The Third Body}

Except Dugan \& Wright (1939) who attributed the period changes to the presence of a third body orbiting the system, Chauby (1980) also reported a third mass of $0.56 \mathrm{M}_{\odot}$ and of period 43.8 yr. Berrington \& Hall (1994) found that the full cycle length of $96 \mathrm{yr}$ when used to calculate the third mass $m_{3}$ resulted in an unreasonably large mass, i.e. $m_{3}=10 \mathrm{M}_{\odot}$. Todoran \& Agerer also used the cycle length of $P=100 \mathrm{yr}$, but obtained again rather a high value for $m_{3}=10.56 \mathrm{M}_{\odot}$. On the other hand, referring again to Figures 2, 6, and 7, the cyclic and wave form of the variations presented in these figure are indicative of some cyclic and regularly changing variations distorted (interrupted) by some other semi-regular changes. Hence, taking into account the above discussion (and non existence of apsidal motion) the most probable causes of such variations 
in the period of this system could be either, the presence of a third body orbiting around the system or magnetic activity cycle modulating the period. Now if one uses the cycle length of $P_{1 \mathrm{mod}}=102.02 \pm 7.4 \mathrm{yr}$, i.e. the period corresponding to the first peak in the Fourier spectrum (Figure 8), then one gets $m_{3}=19 \mathrm{M}_{\odot}$. Moreover, more careful examination of the $\mathrm{O}-\mathrm{C}$ curve (Figure 1) indicates a regular and gradual decrease in the period 1885-1938, followed by an almost constant interval (extremely slow variation) in $P$, from 1940 to 1950 . This interval of constancy may also be observed from the $\mathrm{d} P / \mathrm{d} E$ curve of Figure 5 around the year 1940. After this interval of almost constancy, $P$ begins to increase gradually and regularly up to the year 2003. Taking in to the view just mentioned $P$ variations, and remembering that the estimated semimajor axis $a=52$ a.u. in this work (for the third body) is quite large, I conclude that the third companion receded from the system from the year 1885 to 1940 , reached apastron by the 1940s, and then gradually approached the system by the year 2003, where it appears to be at periastron. Therefore, if we admit a physical meaning to the first peak of the obtained Fourier spectrum, then we should accept a third body with mass $m_{3}=19 \mathrm{M}_{\odot}$, and an orbital radius 52.1 a.u. (estimated in Section 3.3) as a main cause of period change in this system. However, observationally no object of this size has been so far detected nearby to this system. Therefore we are forced to assume that the third mass is a black hole.

The mass of the third body estimated in this work shows rather high value compared to previously estimated values. The reason is that the total mass $\left(m_{1}+m_{2}=3.5 \mathrm{M}_{\odot}\right)$ which I have used in the mass function (i.e. Equation 7) is higher than the other investigators (e.g. FriboesConde \& Herczeg used $\left.m_{1}+m_{2}=1.6 \mathrm{M}_{\odot}\right)$. On the other hand if one uses the other modulating periods, i.e. $P_{2 \mathrm{mod}} \approx 27.87 \pm 2.38 \mathrm{yr}$, mentioned in Section 3, then one gets masses of order $\sim 0.2-0.3 \mathrm{M}_{\odot}$. However the $\mathrm{O}-\mathrm{C}$ amplitude variation is 0.61 days, and this is indicative of a rather large variation in the period of the system. This remarkable changes in period ( $18 \mathrm{~s}$ within less than $75 \mathrm{yr}$ ) were also noticed by Friboes-Conde \& Herczeg (1973) and Berrington \& Hall (1994). It does not seem probable such a small third mass could produce a large variation in the period of the system. Therefore the modulating period 27.87-yr cycles may be attributed to effects of magnetic activity discussed in Section 4.4 below.

\subsection{Mass-Transfer Effects}

SW Cyg is grouped with the kind of stars known as semidetached systems in which the matter from the cool secondary hits the hot primary component. In addition there is strong evidence of mass transfer in Algols including the SW Cyg system, as pointed out thus:

(a) Symmetric appearance and disappearance of red or violet shifted emission features with respect to mid primary eclipse, which according to Struve (1946) are indicators of material in the form of ring or disk around the hotter A2e component.

(b) A small depression in the descending branch of the light curve (between phases $0.80 \& 0.95$ ); see for example Hall \& Garrison (1972); Walter (1971) \& Olson (1987).

(c) Excess of UV radiation (i.e. $(U-B)=0^{m} .3$ for SW Cyg), and the existence of hot spots on this system which were reported by Walter (1971) and Hall \& Garrison (1972). A similar phenomenon was also reported by McNamara \& Feltz (1976) and Manzoori, Jassur, \& Kermani (2006) for other Algols.

(d) There is a remarkable deficiency of carbon and overabundance of nitrogen in the secondaries of Algols as compared to single $\mathrm{G}$ or $\mathrm{K}$ subgiants, as a result of the conversion process of $\mathrm{C}$ to $\mathrm{N}$ via the $\mathrm{CN}$ cycle (Parthasarathy et al. 1983).

Therefore, while considering any physical process in the system, the mass transfer and/or mass loss should also be taken into account. Since period changes in the close binaries are manifestations of geometrical and structural variations, mass transfer and/or mass loss is a related phenomenon as well. If the mass transfer is from the less massive component to the more massive component (i.e. $\Delta m_{2}<0$ ), the period increases, meaning that the size of the orbit (i.e. $a$ ) would widen, and if transfer is from the more massive to the less massive component (i.e. $\Delta m_{2}>0$ ), the orbit would shrink and therefore the period decrease. In our obtained value of $\Delta m_{2}=-7.56 \times 10^{-10} \mathrm{M}_{\odot} \mathrm{yr}^{-1}<0$, the negative sign implies that the effect due to mass transfer alone should be an increase of orbital period of the system.

As stated earlier in Section 3.1, the general trend of $\mathrm{O}-\mathrm{C}$ values displayed in Figure 1 shows an upward curved parabola, roughly fitted by quadratic equation 2 . This overall quadratic character of $\mathrm{O}-\mathrm{C}$ curve suggests a long term secular period increase for the system, which may be attributed to the stable secular mass transfer.

Returning again to the Figure 5 and looking at the form of $\mathrm{d} P / \mathrm{d} E$ function depicted there, I noted that the period rate of change has under gone several changes in sign. This fact exclude the mass transfer alone from the secondary gaint as the explanation for the over all period changes. Since mass transfer is one way process, it could only support the long term secular variation of the $P(E)$ function.

\subsection{The Effect of the Magnetic-Activity Cycle}

It is a well known fact that close binary systems with a cool F-K type stars display enhanced magnetic activity. Furthermore, the tidal interaction between the components in a system forces synchronization of rotational periods of individual stars and orbital period of the system, so that the angular speed increases. Short period Algol binaries i.e. those having orbital period $P<5-6$ days (Richards \& Albright 1993) possesses the above mentioned characteristics, and hence one can expect strong magnetic activity 
in these types of stars due to rapid rotation. Many authors (see e.g. Olson 1981; Hall 1989; Richards 1992, 1993) have discussed the fact that Algol secondaries show a variety of time-dependent magnetic properties, which cause: brightness variations in the light curve (particularly at mid primary eclipse), radiation in X-rays, ultraviolet, and infrared, and cyclic variations in the orbital period of the binary through the Applegate mechanism (briefly explained in Section 2).

One more point which is worth mentioning here is that besides the magnetic activity of the secondary, the primary of this system, i.e. the A2e star, has also been reported to be a magnetically active star (Walter 1971). Therefore both of them have a deflection effect on the plasma ejected by the cool secondary. Walter has mentioned that the absorption is enlarged and the asymmetry of the primary eclipse in light curve is more pronounced due to the motions of ionized particles along magnetic field lines. However, if we accept that both components in this system are magnetically active stars, then the above simple picture of mass transfer effects may not be a correct one, because of magnetic field interactions between the component stars. On the other hand, the simple form of $\mathrm{O}-\mathrm{C}$ curve suggests that there should not be very complicated processes operating in this system. The main reasons for this simple form of $\mathrm{O}-\mathrm{C}$ curve might be the accelerating and localizing effect on the plasma motion due to rather large scale joint $B$ fields $(B=1.3 \mathrm{kG})$, and interaction between magnetic loops attached to the surfaces of two stellar components, which causes the plasma ejected from the secondary to form a regular ring of matter around the primary due to a considerable increase in the stream velocity (velocity of wind) compared to the orbital velocity.

A concluding remark: considering the discussion given above I believe that the most probable causes of period variations in the SW Cyg system are simultaneous actions of two main factors, that are (a) a third body (probably a black hole) orbiting with system and (b) magnetic activity cycles on which are superimposed long term secular mass transfer effects.

\section{Acknowledgments}

I would like to thank Dr. H. Shekari for his careful reading of the first version of this paper and Dr. D. Khojasteh for his wonderful help in converting this paper to Latex format.

\section{References}

Applegate, J. H., 1989, ApJ, 337, 865

Applegate, J. H., 1992, ApJ, 385, 621

Applegate, J. H. \& Patterson, J., 1987, ApJ, 322, L99

Berrington, R. C. \& Hall, D. S., 1994, AJ, 107, 1868

Blazko, S., 1924, ANZi, 7, 35

Bondi, H. \& Hoyle, F., 1944, MNRAS, 104, 273

Chaubey, U. S., 1980, AP\&SS, 103, 55

Dugan, R. S. \& Wright, F. W., 1939, Contribution from the Prinstone University Observatory

Frieboes-Conde, H. \& Herczeg, T., 1973, A\&AS, 12, 1

Hall, D. S., 1989, SSRV, 50, 219

Hall, D. S., 1991, ApJ, 380, L85

Hall, D. S. \& Garrison, L. M., Jr., 1972, PASP, 84, 552

Hall, D. S. \& Kreiner, J., 1980, AcA, 30, 387

Hack, M., 1984, IUE Proposal, 1944

Huang, S.-S., 1963, ApJ, 138, 417

Irwin, J. B., 1959, AJ, 64, 149

Kalimeris, A., Livaniou, H. R. \& Rovithis, P., 1994, A\&A, 282,775

Lanza, A. F., Rodono, M. \& Ronse, R., 1998, MNRAS, 296, 893

Manzoori, D., Jassur, D. M. Z. \& Kermani, M. H., 2006, Ap\&SS, 302,145

McNamara, D. H. \& Feltz, K. A., Jr., 1976, PASP, 88, 688

Olson, E. C., 1981, ApJ, 250, 704

Olson, E. C., 1985, PASP, 97, 731

Olson, E. C., 1987, AJ, 94, 1043

Olson, E. C. \& Etzel, P. B., 1993, AJ, 106, 342

Parthasarathy, M., Lambert, D. L. \& Tomkin, J., 1983, MNRAS, 203, 1063

Qian, S. B., Zhu, L. Y., \& Boonrucksar, S., 2002, A\&A, 396, 609

Richards, M. T., 1992, ApJ, 387, 329

Richards, M. T., 1993, ApJS, 86, 255

Richards, M. T. \& Albright, G. E., 1993, ApJS, 88, 199

Simon, V., 1997, A\&A, 327, 1087

Struve, O., 1946, ApJ, 104, 253

Todoran, C. N. \& Agerer, Z., 1994, AN, 315, 349

Walter, K., 1971, A\&A, 13, 249

Wendell, O. C., 1909, AnHar, 69, 1 